\title{
Engineering Neurospora crassa for cellobionate production directly from cellulose without
}

\section{any enzyme addition}

\author{
Hui Lin ${ }^{1}$, Amanda Hildebrand ${ }^{1}$, Takao Kasuga ${ }^{2,3}$, and Zhiliang Fan ${ }^{1 *}$
}

${ }^{1}$ Biological and Agricultural Engineering Department and ${ }^{2}$ Department of Plant Pathology, University of California, Davis, One Shields Avenue, Davis, CA 95616; ${ }^{3}$ United States

Department of Agriculture-Agricultural Research Service, Davis, CA 95616

Running title: cellobionate production by N. crassa

Correspondence: Zhiliang Fan, Tel: 530-754-0317; Fax: 530-752-2640;

E-mail: jzfan@ucdavis.edu

Keywords: cellobionate, cellobiose dehydrogenase, laccase, Neurospora crassa

\begin{abstract}
In a cellulosic biorefinery, the cellulase enzymes needed for hydrolysis are one of the major contributors to high processing costs, while the hydrolysis product, cellobiose, has strong inhibition to the cellulases. In this study, we report engineering recombinant Neurospora crassa strains which are able to produce cellobionate, an organic acid, from cellulose without any enzyme addition. Recombinant strains were constructed by heterologously expressing laccase

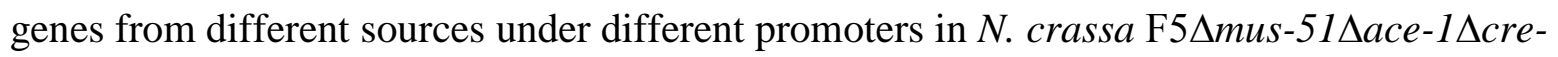
$1 \Delta n d v B$ which has six out of seven $\beta$-glucosidase (bgl), two transcription factor (crel and ace-l), and the cellobionate phosphorylase $(n d v B)$ genes deleted. The strain expressing laccase from Botrytis aclada under a copper metallothionein promoter (HL10) produced the highest laccase activity. N. crassa HL10 produced $47.4 \mathrm{mM}$ cellobionate from cellulose without any enzyme
\end{abstract}


addition. The yield of cellobionate from hydrolyzed cellulose was about $94.5 \%$. Conversion of cellobiose to cellobionate improved cellulose conversion and increases product yield. 


\section{$1 \quad$ 1.Introduction}

2 The microbiological production of organic acids is an attractive alternative to chemical

3 synthesis. The native and genetically engineered microorganism microbial have been widely

4 used in the production of a variety of organic acids such as acetic acid, gluoncic acid, lactic acid

5 etc [1]. In order to compete with petroleum-based processes for the production of carboxylic

6 acids, the development of microbial processes utilizing low-cost substrates is essential [1].

7 Cellulosic biomass is a sustainable, low cost, and abundant feedstock for organic fuels and

8 chemicals production. However, the major impediment for the commercialization of cellulosic

9 bioprocessing is the lack of a low-cost processing technology [2]. Most of cellulosic biorefineries

10 for organic acids production need cellulases addition to hydrolyze cellulosic biomass to

11 fermentable sugars, which presents a substantial processing cost.

12 In this study, we report engineering a filamentous fungus, Neurospora crassa, for the

13 production of a specialty organic acid, cellobionic acid, directly from cellulose without any

14 enzyme addition. The chemical structure of cellobionic acid comprises a glucose moiety linked

15 to a gluconic acid molecule. It is an aldonic acid obtained from the oxidation of cellobiose.

16 Although there are few reports in literature exploring the application of cellobionic acid, its

17 stereoisomer lactobionic acid has numerous applications in pharmaceutical, food, and cosmetics

18 industries due to its unique physiochemical properties [3]. Cellobionic acid is expected to have

19 similar applications due to the similar physiochemical properties.

20 In a prior study, the strain N. crassa F5 $2 m u s-51$ was constructed by deleting six out of

21 seven of its $\beta$-glucosidase genes and a gene involved in non-homologous end joining from the

22 wild type $N$. crassa FGSC 2489 that produces a full array of cellulases and cellobiose

23 dehydrogenases. The strain $N$. crassa F5 $2 m u s-51$ was able to produce about $20 \mathrm{mM}(7 \mathrm{~g} / \mathrm{L})$ 
24 cellobiose and less than $1 \mathrm{mM}$ of cellobionic acid from $20 \mathrm{~g} / \mathrm{L}$ Avicel in 4 days [4]. In order to

25 further improve the production of cellobiose and its derivatives, we deleted two transcription

26 factor genes (cre-1 and ace-1) involved in carbon catabolite repression, and a gene encoding a

27 cellobionate phosphorylase gene $(n d v B)$, which is necessary for cellobionate utilization, resulting

28 strain $N$. crassa F5 $\Delta m u s-51 \Delta a c e-1 \Delta c r e-1 \Delta n d v B$. This strain was able to produce $20 \mathrm{mM}$ (7

$29 \mathrm{~g} / \mathrm{L})$ cellobiose and $10 \mathrm{mM}$ cellobionate $(3.5 \mathrm{~g} / \mathrm{L})$ from $20 \mathrm{~g} / \mathrm{L}$ Avicel in 7 days [5].

Product inhibition of cellulases by its hydrolysis product cellobiose has been known to

31 significantly decrease the rates of enzymatic hydrolysis and limits the sugar yields in cellulase

32 mediated hydrolysis processes [5, 6]. Alleviating product inhibition is therefore essential for

33 improving the process efficiency. Since cellobionic acid is a much weaker inhibitor to

34 cellobiohydrolase I as compared to cellobiose[5], conversion of cellobiose to cellobionic acid

35 could alleviate product inhibition on cellulases, therefore, increasing cellulose hydrolysis rate

36 and improving product yield. In the native system of $N$. crassa, cellobiose dehydrogenase

37 (CDH) catalyzes the oxidation of cellobiose to cellobionate. However, the re-oxidization of the

38 reduced $\mathrm{CDH}$ by a polysaccharide monooxygenase and eventually oxygen was the rate limiting

39 step, which led to slow conversion of cellobiose to cellobionate [7]. One strategy to increase the

40 rate of cellobiose oxidation by $\mathrm{CDH}$ with oxygen as the final electron acceptor is to employ

41 catalytic amounts of redox mediator with in situ regeneration of the redox mediator by laccase

42 using the CDH-redox-laccase system [8]. In such a system, cellobiose is oxidized to cellobionic

43 acid, in turn, the reduced $\mathrm{CDH}$ is re-oxidized with the help of a redox mediator, which is then

44 regenerated through oxidation by laccase. Lastly, laccase is regenerated when the electrons are

45 passed to oxygen, the final electron acceptor. The addition of catalytic amount of laccase (0.05

$46 \mathrm{U} / \mathrm{mL}$ ) and 2,2'-azino-bis (3-ethylbenzothiazoline-6-sulfonic acid (ABTS) $(0.01 \mathrm{mM}$ ) as the 


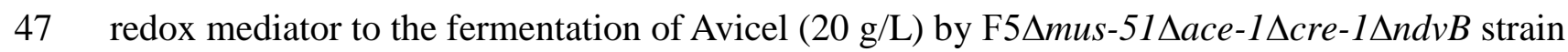

48 produced about $40 \mathrm{mM}$ cellobionate after 120 hours. Whereas, the fermentation using the same

49 strain without laccase and ABTS addition only $10 \mathrm{mM}$ cellobionate and about $20 \mathrm{mM}$ of

50 cellobiose remain un-converted [8].

51 Since exogenous enzymes addition remains one of the most expensive components of a

52 biochemical conversion process, it is desirable to develop $N$. crassa strains that do not require

53 any exogenous enzyme addition for the production of cellobionate directly from cellulose. In this

54 study, the N. crassa laccase gene (Nclac) and a codon optimized Botrytis aclada laccase gene

55 (sBalac, GenBank: JN559771) were expressed under the control of the constitutive Aspergillus

56 nidulans glyceraldehyde-3-phosphate dehydrogenase (gpdA) promoter, a long copper

57 metallothionein promoter fragment $(\mathrm{cmt})$, and a short copper metallothionein promoter fragment

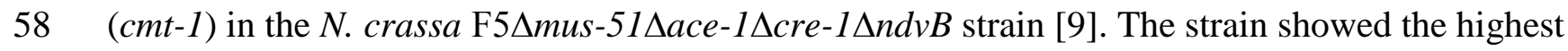

59 laccase activities when $c m t$ or $c m t-1$ was used as the promoter to express sBalac. Cellobionate

60 was produced from cellulose by the strain HL10, which expresses sBalac under the control of

61 cmt-1, without any enzyme addition.

62

\section{2. Material and Methods}

\section{$64 \quad 2.1$ Strains and materials}

65 Strains used in this study and their sources are listed in Table 1. Primers used in strain 66 construction and verification are listed in Table 2. The strain F5 $2 m u s-51 \Delta a c e-1 \Delta c r e-1 \Delta n d v B$ 67 was constructed in a previous study [10]. The methods for constructing AH003, HL 04, 05, 10, 6811 and 13 and verifying them are described in the following sections. ABTS was purchased from 
69 Roche Diagnostics (Indianapolis, IN). All other chemicals were purchased from Sigma-Aldrich.

70 All the primers used for strain construction are shown in Table 1S.

72 2.2Construction of vectors

73 Plasmid pCSR1-gpdA-Nclac was constructed to express $N$. crassa laccase under the 74 Aspergillus nidulans glyceraldehyde-3-phosphate dehydrogenase (gpdA) promoter in N. crassa 75 F5 $4 m u s-51 \Delta c r e-1 \Delta a c e-1 \Delta n d v B$. The 1917 bp N. crassa laccase gene Nclac was amplified with 76 primers (Nclac-F and Nclac-R contained PmlI and SnaBI restriction site, respectively) from the

77 N. crassa FGSC2489 genomic DNA. Then, the PCR product was digested by PmlI and SnaBI 78 and cloned into the pCSR1-cdh1 vector with the $c d h 1$ gene cut out by the same two enzymes 79 [11] [12], building the plasmid pCSR1-gpdA-Nclac. The constructed plasmid contained the Nclac 80 gene flanked by a $g p d A$ promoter and an $A$. nidulans trpC terminator.

Plasmid pCSR1-gpdA-sBalac was constructed with a similar method in order to express

83 laccase from B. aclada under the gpdA promoter. The fragment containing the codon optimized

84 B. aclada laccase gene, a 6-His tag (sBalac, GenBank: JN559771), and PmlI and SnaBI

85 restriction sites was synthesized by Invitrogen (Thermo Fisher Scientific, CA). The product was

86 then digested by PmlI and SnaBI and cloned into pCSR1-cdh1 vector with $c d h-1$ gene cut out,

87 building the plasmid pCSR1-gpdA-sBalac.

Plasmids pCSR1-pcmt-Nclac and pCSR1-pcmt-sBalac were constructed to express

90 laccase from N. crassa or B. aclada under the copper metallothionein (cmt) promoters [13]. The $914480 \mathrm{bp}$ copper metallothionein $(\mathrm{cmt})$ promoter was amplified from the genomic DNA of $N$. 
92 crassa FGSC 2489 through PCR using primers (pcmt-slic-F and pemt-slic-NcR, or pemt-slic-F

93 and pcmt-slic-sBaR). The pCSR1-gpdA-Nclac and pCSR1-gpdA-sBalac vector were amplified

94 through PCR using primers (NcLac_F and 3flank_slic_R, and sBalacF and 3flank_slic_R,

95 respectively), and then pcmt PCR product was ligated into the vector PCR product by sequence

96 and ligation independent cloning (SLIC) [14], building the plasmid pCSRI-pmct-Nclac and

97 pCSR1-pcmt-sBalac.

98

99 Plasmids pCSR1-pmct1-Nclac and pCSR1-pcmt1-sBalac were constructed using similar

100 methods in order to express laccase from N. crassa or B. aclada under a truncated copper

101 metallothionein ( $\mathrm{cmt}$ ) promoter [13]. The $511 \mathrm{bp}$ copper metallothionein ( $\mathrm{cmt}-1)$ promoter was

102 amplified with primers (pemt1-slic-F and pcmt-slic-NcR, or pemt1-slic-F and pcmt-slic-sBaR)

103 from $N$. crassa FGSC2489 genomic DNA. The pCSR1-gpdA-Nclac and pCSR1-gpdA-sBalac

104 vector was amplified through PCR using primers (NcLac_F and 3flank_slic_R, and sBalacF and

105 3flank_slic_R, respectively), and then the pcmt PCR product was ligated into the vector PCR

106 product by sequence and ligation independent cloning (SLIC), building the plasmid pCSR1-

107 pmct1-Nclac and pCSR1-pcmt1-sBalac.

108

109 2.3. Transformation and diagnosis

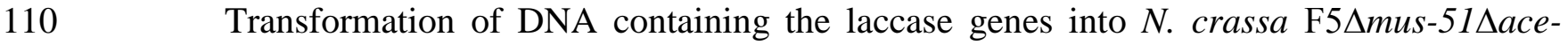

$1111 \Delta c r e-1 \Delta n d v B$ and screening of the transformants were performed following the protocols

112 reported by Bardiya and Shiu [12]. Plasmids were amplified in E. coli Top10, and purified using

113 a Zyppy ${ }^{\mathrm{TM}}$ Plasmid Miniprep Kit. The plasmids were double digested by PciI and PsiI, purified

114 by agarose gel and extracted by NucleoSpin Gel Clean-up Kit (MACHEREY-NAGEL, 
115 Germany). $90 \mu \mathrm{L}$ conidia suspension $\left(2.5 \times 10^{9}\right.$ conidia/mL) and $10 \mu \mathrm{L}$ DNA solution (100 $116 \mathrm{ng} / \mu \mathrm{L})$ were mixed in microcentrifuge tubes $(1.5 \mathrm{~mL})$ on ice for $5 \mathrm{~min}$, then transferred to pre-

117 chilled 0.2-cm electroporation cuvette (BioRad, Richmond, CA). After shock at $1.5 \mathrm{kV} / \mathrm{cm} ; 25$

$118 \mathrm{uFD}$ and $600 \mathrm{ohms}, 1 \mathrm{~mL}$ pre-chilled recovery solution containing $1 \mathrm{M}$ sorbitol, $2 \%$ yeast extract 119 and $20 \mathrm{~g} / \mathrm{L}$ sucrose was immediately added in each cuvette, and transferred into a $15 \mathrm{~mL}$ conical 120 tube. The cells were cultured at $30^{\circ} \mathrm{C}$ and shaken at $150 \mathrm{rpm}$ for 6 hours, then spread on 121 selection plates $(1 \times$ Vogel's, $2 \%$ yeast extract, $20 \mathrm{~g} / \mathrm{L}$ sorbose, $0.5 \mathrm{~g} / \mathrm{L}$ fructose, $0.5 \mathrm{~g} / \mathrm{L}$ glucose,

$1225 \mu \mathrm{g} / \mathrm{mL}$ cyclosporine A, 2\% agar). The positive transformants resistant to cyclosporine A were 123 screened by diagnostic PCR.

\section{$125 \quad$ 2.4. Fermentation experiments}

$50 \mathrm{~mL} 1 x$ Vogel's media containing glucose $(40 \mathrm{~g} / \mathrm{L})$ or Avicel $(20 \mathrm{~g} / \mathrm{L})$ and $\mathrm{CuSO}_{4}(0.8$

$127 \mathrm{mM}$ ) in $250 \mathrm{~mL}$ Erlenmeyer flasks were inoculated by 10-14 day old conidia suspensions of the 128 recombinant $N$. crassa strains at a final $\mathrm{OD}_{420}$ of 0.05 [15]. Flasks were incubated at $28^{\circ} \mathrm{C}$ and 129 shaken at $200 \mathrm{rpm}$ with constant light. At the beginning of each fermentation, $3 \mathrm{~g} / \mathrm{L}$ of glucose 130 was added to initiate conidial germination when Avicel was used as the substrate, and the $\mathrm{pH}$ of 131 the cultures was adjusted to around $\mathrm{pH}$ 5.0-5.5 by $\mathrm{NaOH}(1 \mathrm{M})$. Samples were taken at various 132 time intervals for enzyme activity analysis and compositional analysis.

\section{2.5. Sample analysis}

135 Concentrations of sugars (glucose, cellobiose, cellobionate) were analyzed using a 136 Shimadzu HPLC equipped with a refraction index detector (RID), PDA detector, and a 
137 CARBOSep COREGEL-87C (Transgenomic, San Jose, CA, USA) column. 4 mM calcium 138 chloride at a flow rate of $0.5 \mathrm{~mL} / \mathrm{min}$ was used as the mobile phase.

\subsection{Enzyme activity assay}

Laccase activity assays were performed in 96-well plate following previous protocol with 142 minor modifications [8]. The reaction mixture contained $180 \mu \mathrm{L}$ of $1 \mathrm{mM}$ ABTS in $100 \mathrm{mM}$ 143 sodium acetate buffer, $\mathrm{pH}$ 4.5. One unit of laccase activity is defined as the amount of enzyme 144 oxidizing $1 \mu \mathrm{mol}$ of ABTS per minute under the above reaction conditions.

145 Exoglucanase activity assay was performed using $p$-nitrophenyl- $\beta$-D-lactopyranoside 146 (pNPL, Sigma) as the substrate following previous a protocol [9]. The reaction mixtures 147 containing $100 \mathrm{uL}$ of $1 \mathrm{mg} / \mathrm{mL}$ pNPL in $50 \mathrm{mM}$ citric acid buffer (pH 5.0) and $80 \mathrm{uL}$ of sample 148 were incubated for 15 minutes at $37{ }^{\circ} \mathrm{C}$. The amount of released $p$-nitrophenol was measured at $149405 \mathrm{~nm}$ after addition of $120 \mathrm{uL}$ of $1 \mathrm{M} \mathrm{NaOH}$. One unit of enzyme activity is defined as the 150 amount of enzyme required to release 1 umole of $p$-nitrophenol per minute.

\subsection{Mycelial biomass measurements}

The mycelia mass in the fermentation samples was measured by extracting ergosterol

154 from the mycelia and quantification by HPLC following a protocol described by Hildebrand et al 155 [8]. The amount of biomass was quantified using a standard curve prepared with known $N$. 156 crassa dry mycelia.

\section{Results}


Six expression cassettes carrying the A. nidulans gpdA promoter, the $N$. crassa

161 FGSC2489 $\mathrm{cmt}$ (copper metallothionein, $4.5 \mathrm{~kb}$ fragment) promoter, or the $N$. crassa FGSC2489

162 cmt1 (copper metallothionein, $511 \mathrm{bp}$ fragment) promoter; the $N$. crassa FGSC2489 laccase gene

163 (Nclac), or the synthesized codon-optimized B. aclada laccase gene (sBalac); and the A. nidulans

$164 \operatorname{trp} C$ terminator were knocked in the $c s r-1$ site of $N$. crassa F5 $\Delta m u s-51 \Delta a c e-1 \Delta$ cre-1 $\Delta n d v B$ for

165 laccase over-expression. Correct transformants were confirmed by diagnostic PCR.

\section{3.2. Laccase production by recombinant strains on glucose}

The laccase activities produced by the recombinant strains were characterized after 3

169 days' culture in Vogel's media containing $40 \mathrm{~g} / \mathrm{L}$ glucose and $0.8 \mathrm{mM} \mathrm{CuSO}$. As shown in

170 Figure 1, N. crassa HL10, expressing sBalac under cmt-1 promoter, and $N$. crassa HL13, 171 expressing sBalac under $c m t$ promoter, were the most successful laccase producers. Both strains 172 secreted up to $0.59 \mathrm{U} / \mathrm{mL}$ laccase in the broth at day 3. The strain AH003, which expressed 173 Nclac under the gpdA promoter, did not show obvious activity. Other strains produced detectable

174 laccase activity (0.03-0.08 U/mL). Overall, Nclac or sBalac expressions under $N$. crassa cmt1 or 175 cmt promoter achieved 10-fold higher activities than those under the A. nidulans gpdA promoter. 176 Strains expressing sBalac produced higher laccase activity as compared to the strain expressing 177 Nclac when the same promoter was used. The two N. crassa recombinants (HL10 and HL13) 178 that express the codon optimized B. aclada laccase gene under the $4.5 \mathrm{~kb}$ fragment or $511 \mathrm{bp}$ 179 fragment of $\mathrm{cmt}$ promoter were the best laccase producers. They were chosen for further study. 
183 cmt1 promoter, copper ions likely play an important role in laccase activity and expression [13,

184 16]. Vogel's medium contains only $1 \mu \mathrm{M}$ copper, and it was unknown if this was high enough to

185 induce the $c m t$ or $c m t l$ promoter and express functional laccase. Here, the effects of copper 186 concentrations and its addition time on laccase production of $N$. crassa HL10 and HL13 were 187 investigated (Figure 2 and Figure 3). $\mathrm{CuSO}_{4}$ was added to a final concentration of $0.5 \mathrm{mM}$ at 0 , 18812,24 , or 36 hours for inducing the $c m t$ or $c m t 1$ promoter and functional laccase expression 189 (Figure 2). The laccase production profiles were not affected by the copper addition time. When 190 copper was added at 0,12 , or 24 hours, all strains produced the maximum laccase activities at 60 191 hours (Figure 2). However, a little less laccase was produced when $\mathrm{CuSO}_{4}$ was added at 36 192 hours as compared to the cases when the copper solution was added earlier.

193 The effects of copper concentrations on laccase expression in N. crassa HL10 and HL13

194 were also investigated. As shown in Figure 3, no obvious laccase production was detected $195(<0.03 \mathrm{U} / \mathrm{mL})$ by either HL10 or HL13 when the culture contained less than $0.1 \mathrm{mM} \mathrm{CuSO}_{4}$ 196 (Figure 3). When the copper concentrations in media were increased to $0.2 \mathrm{mM}$ or more, HL10 197 and HL13 produced obvious laccase activity in the media. Laccase production by HL10 and 198 HL13 were tightly related to the copper concentrations in the media. Both strains were 199 responsive to copper concentrations in the broth and their response patterns are similar to each 200 other. Strain N. crassa HL10 was chosen for further study.

\section{3.4. The effect of temperature on laccase production}

202 Temperature is an important factor in N. crassa protein expression [17]. To evaluate the 203 effect of temperature on laccase production by the recombinant strains, $N$. crassa HL10 was 204 cultured on glucose $(40 \mathrm{~g} / \mathrm{L})$ in Vogel's media with $0.8 \mathrm{mM} \mathrm{CuSO}_{4}$ at different temperatures. As 
205 shown in Figure 4, very little laccase was produced when N. crassa HL10 was cultured at 34

$206{ }^{\circ} \mathrm{C}$. When the culture temperature lowered to 24 or $26{ }^{\circ} \mathrm{C}$, the laccase production reached the

207 highest level of about $0.98 \mathrm{U} / \mathrm{mL}$. Lower temperatures seem to favor the laccase production,

208 which is a phenomena seen in laccase heterologous expression in Pichia as well [18]. Whether

209 the temperature affect the protein expression seems to be protein specific [18].

210

211 3.5. The effect of temperature on cellobionate production

212 The effect of temperature on cellobionate production by $N$. crassa HL10 was tested and

213 the results are shown in Figure 5. N. crassa HL10 produced the highest concentration of

214 cellobionate $(48 \mathrm{mM})$ when the culture was incubated at $30{ }^{\circ} \mathrm{C}$, while the concentration of

215 cellobionate produced was lowest $(30 \mathrm{mM})$ when the culture was incubated at $24{ }^{\circ} \mathrm{C}$. The trend 216 was opposite that of laccase production when the strain was grown on glucose.

3.6 N. crassa F5 4mus-514ace-1Acre-14ndvB and $N$. crassa HL10 fermentation on Avicel

N. crassa F5 mus-514ace-1 14 cre-1 $1 \Delta n d v B$ and $N$. crassa HL10 were grown on Avicel in

220 Vogel's medium. ABTS $(0.1 \mathrm{mM})$ was added into the fermentations at day 3. N. crassa HL10

221 produced higher exoglucanase activities than those of $N$. crassa F5 mus-51 1 ace-1 14 cre-1 $\Delta$ ndvB

222 cultures in the fermentation (Figure 6A). Cellobionate production by N. crassa HL10 increased

223 over the course of 8 days of fermentation, and cellobionate concentration reached $47.4 \mathrm{mM}$,

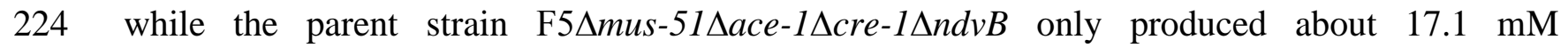

225 cellobionate (Figure 6B). There was only about $0.6 \mathrm{mM}$ cellobiose remaining in the

226 fermentation broth for the HL10 strain, compared to $20.3 \mathrm{mM}$ cellobiose for the F5

$22751 \Delta$ ace-1 $1 \Delta c r e-1 \Delta n d v B$ strain. The HL10 strain produced $28.3 \%$ more of combined cellobiose 


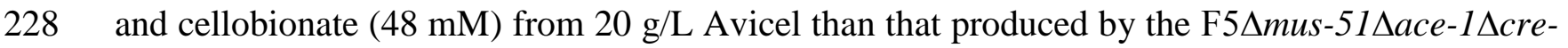

$229 \quad 1 \Delta n d v B$ strain $(37.4 \mathrm{mM})$.

230 In a separate experiment conducted identically to the above experiment, flasks were

231 harvested after an 8-day fermentation and analyzed for residual Avicel and mycelium amount.

232 As shown in Table 3, HL10 has higher cellulose conversion as compared to N. crassa F5 Aace-

$2331 \Delta c r e-1 \Delta n d v B$. However, it produced slightly less mycelial biomass.

\section{Discussion}

Wild type $N$. crassa contains a laccase gene in its genome, which is transcribed only

237 during sexual differentiation stage [19]. During vegetative growth, the laccase gene is repressed

238 unless some protein synthesis inhibitors such as cycloheximide are added to trigger its

239 expression [20]. Homologous expression of laccase in $N$. crassa under a $3.6 \mathrm{~kb}$ cmt promoter to

240 enable its expression in the vegetative growth phase was achieved by Schilling et al [16 ]. We

241 achieved the expression of Nclac and sBalac under the control of a $4.5 \mathrm{~kb} c m t$ promoter which

242 contains the $3.6 \mathrm{~kb} \mathrm{cmt}$ promoter and enabled laccase production under vegetative growth phase

243 as well. The transgenes in the strains prepared by Schilling et al., are most likely to be

244 ectopically integrated in a random genome location through non-homologous recombination

245 [16]. In contrast, our engineered strains were prepared by integrating a single copy of a laccase

246 expression cassette at the $\operatorname{csr}-1$ locus through homologous recombination and the resulting strain

247 is a homokaryotic thanks to improved genetic engineering tools. Despite of this difference, our

248 strain and the strain reported by Schilling et al. behaved similarly in response to available

249 copper ions. Laccase production was only achievable when copper was added. The extracellular 
250 laccase levels became detectable 36 hours after inoculation and the maximal laccase activities 251 were detected around 60 hours.

252 The $c m t$ promoter is an ideal promoter for regulated protein expression in $N$. crassa due 253 to its high inducibility, ease of use, and economic applicability [21]. Kupper et al. compared a 254 full length $3.6 \mathrm{~kb}$ and a truncated $400 \mathrm{bp}$ promoter to express tyrosinase in $N$. crassa to enable 255 the production of tyrosine under vegetative growth phase [13]. Recombinant strains using both 256 promoters successfully produced tyrosinase activities. They reported that the full length $3.6 \mathrm{~kb}$ 257 promoter was found to be inducible, while the $400 \mathrm{bp}$ version was constitutive. In this study, we 258 achieved the expression of laccases under the control of a long $4.5 \mathrm{~kb} \mathrm{cmt}$ promoter and a shorter $259511 \mathrm{bp} c m t-1$ promoter. The $4.5 \mathrm{~kb} \mathrm{cmt}$ promoter used in our study contains the $3.6 \mathrm{~kb}$ promoter 260 reported by Tupper et al. Likewise, our 511 bp promoter contains their 411 bp promoter. They 261 were built a little longer for the convenience of cloning. The $511 \mathrm{bp}$ promoter is located 262 upstream (from -511 to -1) of the copper metallothionein gene NCU05561. To our surprise, the 263511 bp promoter was found to be inducible instead of constitutive. The strains containing either 264 the long or the short promoters behave very similar to each other. The $511 \mathrm{bp}$ promoter is likely 265 to contain all the key elements to function as efficiently as the $4.5 \mathrm{~kb}$ one. It contained a typical 266 TATA box at site -176 [22], and two putative CCAAT boxes at position -473 and -375 [23].

267 We also tested a constitutive promoter from A. nidulans's D-glyceraldehyde-3-phosphate 268 dehydrogenase gene ( $g p d A)$ for laccase production in N. crassa. Although the gpdA promoter has 269 been successfully used as a strong promoter for heterologous gene expression in fungi [24], 270 including expression of cellobiose dehydrogenase in $N$. crassa by our lab[11], we observed 271 laccase (lac) expression under the gpdA promoter in N. crassa F5 $1 m u s-51 \Delta a c e-1 \Delta$ cre-1AndvB 272 was inferior to those under $c m t$ or $c m t-1$ control. 
274 less laccase activity than $N$. crassa HL10 which expresses sBalac. A possible reason is that $N$. 275 crassa laccase has lower catalytic activity than that of B. aclada laccase on ABTS [9, 20] or the 276 expression level of $N$. crassa laccase was lower.

The laccase production decrease when the culture temperature increases in the range of

278 24-30 ${ }^{\circ} \mathrm{C}$ when the recombinant strains were grown on glucose. However, the cellobionate 279 production was highest at $30^{\circ} \mathrm{C}$ in the Avicel fermentation. Since the cellobionate production 280 from Avicel needs the coordination of cellulases, cellobiose dehydrogenase, and laccases, the 281 overall reaction seemed to favor a high temperature instead of a low temperature. Since 282 cellobiose was present in the whole process, it indicates that the oxidation reaction was the rate 283 limiting step.

284 When $N$. crassa HL10 and N. crassa F54mus-514ace-1Acre-1AndvB both grew on 20 $285 \mathrm{~g} / \mathrm{L}$ Avicel in Vogel's medium containing $3 \mathrm{~g} / \mathrm{L}$ glucose, $0.1 \mathrm{mM}$ ABTS and $0.8 \mathrm{mM} \mathrm{CuSO}$, 286 HL10 produced cellobionate as the main product $(47.4 \mathrm{mM})$ with only about $0.6 \mathrm{mM}$ of 287 cellobiose unconverted, while the strain F5 $1 m u s-51 \Delta a c e-1 \Delta c r e-1 \Delta n d v B$ produced cellobiose $288(55 \%)$ as the major product and cellobionate as the minor product (45\%). The $N$. crassa HL10 289 achieved higher cellulose conversion than that of $N$. crassa F5 $4 m u s-51 \Delta a c e-1 \Delta c r e-1 \Delta n d v B$, 290 which indicated that accelerating of the conversion of cellobiose to cellobionate is an effective 291 strategy to alleviate cellobiose inhibition on cellulases and to improve celluobionate yield.

292 Filmentous fungi have been widely known for their ability to degrade cellulosic biomass 293 and for their ability to convert sugars to organic acids. To our knowledge, this study was the first 294 report to exploit both the cellulose degradation and organic acid production properties of a single 295 filmanetous fungi. It is worth noting that cellobionate could potentiall be a high value chemical 
296 with wide application itself. It could also be alternative carbon source instead of cellobiose or

297 glucose for fuel and chemical production [5]. It can be directly used by engineered Escherichia

298 coli for isobutanol production and an engineered Saccharomyces cerevisiae for ethanol

299 production $[25,26]$. Both of its hydrolysis products, glucose and gluconate, could be used for

300 ethanol and acetic acid production by E. coli [4, 27].

301

302 5. Conlcusions

303 In conclusion, we created an engineered N. crassa strain, HL10, for conversion of 304 cellulose to cellobionate without any enzyme addition. N. crassa HL10 achieved higher cellulose 305 conversion and higher product yield than those of its parent strain, which illustrates the 306 advantages of relieving product inhibition by conversion of cellobiose to cellobionate in 307 cellulose hydrolysis. This strain has the potential to be used for homo-cellobionate production 308 from cellulosic biomass.

309

\section{Acknowledgement}

311 This project was supported by Agriculture and Food Research Initiative Competitive 312 Grant No. 2011-67009-20060 from the USDA National Institute of Food and Agriculture. The 313 authors thank Eric Walters for reading through the paper. 
316 [1] Nigam PS. Production of organic acids from agro-industrial residues. 1st ed: Netherlands: 317 Springer; ; 2009.

318 [2] Lynd LR, Wyman CE, Gerngross TU. Biocommodity Engineering. Biotechnol Prog. 319 1999;15:777-93.

320 [3] Alonso S, Rendueles M, Diaz M. Bio-production of lactobionic acid: current status, applications and future prospects. Biotechnol Adv. 2013;31:1275-91.

[4] Fan Z, Wu W, Hildebrand A, Kasuga T, Zhang R, Xiong X. A novel biochemical route for fuels and chemicals production from cellulosic biomass. PloS one. 2012;7:e31693.

[5] Hildebrand A, Addison JB, Kasuga T, Fan ZL. Cellobionic acid inhibition of cellobiohydrolase I and cellobiose dehydrogenase. Biochemical Engineering Journal. 2016;109:236-42.

[6] Andric P, Meyer AS, Jensen PA, Dam-Johansen K. Reactor design for minimizing product inhibition during enzymatic lignocellulose hydrolysis: II. Quantification of inhibition and suitability of membrane reactors. Biotechnology advances. 2010;28:407-25.

[7] Beeson WT, Phillips CM, Cate JHD, Marletta MA. Oxidative Cleavage of Cellulose by Fungal Copper-Dependent Polysaccharide Monooxygenases. J Am Chem Soc. 2012;134:890-2.

[8] Hildebrand A, Kasuga T, Fan ZL. Production of Cellobionate from Cellulose Using an Engineered Neurospora crassa Strain with Laccase and Redox Mediator Addition. PloS one. $2015 ; 10$.

[9] Kittl R, Mueangtoom K, Gonaus C, Khazaneh ST, Sygmund C, Haltrich D, et al. A chloride tolerant laccase from the plant pathogen ascomycete Botrytis aclada expressed at high levels in Pichia pastoris. J Biotechnol. 2012;157:304-14.

[10] Hildebrand A, Szewczyk E, Lin H, Kasuga T, Fan ZL. Engineering Neurospora crassa for Improved Cellobiose and Cellobionate Production. Appl Environ Microbiol. 2015;81:597-603.

[11] Zhang R, Xu C, Shen Q, Kasuga T, Wu W, Szewczyk E, et al. Characterization of two cellobiose dehydrogenases and comparison of their contributions to total activity in Neurospora crassa. Int Biodeterior Biodegrad. 2013;82:24-32.

[12] Bardiya N, Shiu PKT. Cyclosporin A-resistance based gene placement system for Neurospora crassa. Fungal Genet Biol. 2007;44:307-14.

[13] Kupper U, Linden M, Cao KZ, Lerch K. Expression of tyrosinase in vegetative cultures of Neurospora crassa transformed with a metallothionein promoter/protyrosinase fusion gene. Curr Genet. 1990;18:331-5.

[14] Jeong JY, Yim HS, Ryu JY, Lee HS, Lee JH, Seen DS, et al. One-Step Sequence- and Ligation-Independent Cloning as a Rapid and Versatile Cloning Method for Functional Genomics Studies. Applied and Environmental Microbiology. 2012;78:5440-3.

[15] Hildebrand A, Kasuga T, Fan Z. Production of cellobionate from cellulose using an engineered Neurospora crassa strain with laccase and redox mediator addition. Plos One. 2015;10:e0123006.

[16] Schilling B, Linden RM, Kupper U, Lerch K. Expression of Neurospora crassa laccase under the control of the copper-inducible metallothionein promoter. Curr Genet. 1992;22:197203.

[17] Romero MD, Aguado J, Gonzalez L, Ladero M. Cellulase production by Neurospora crassa on wheat straw. Enzyme Microb Technol. 1999;25:244-50.

[18] Park M, Kim M, Kim S, Ha B, Ro HS. Differential Expression of Laccase Genes in Pleurotus ostreatus and Biochemical Characterization of Laccase Isozymes Produced in Pichia pastoris. Mycobiology. 2015;43:280-7.

[19] Scherer M, Wei HJ, Liese R, Fischer R. Aspergillus nidulans catalase-peroxidase gene (cpeA) is transcriptionally induced during sexual development through the transcription factor StuA. Eukaryot Cell. 2002;1:725-35. 
364 [20] Froehner SC, Eriksson KE. Purification and Properties of Neurospora-Crassa Laccase. J 365 Bacteriol. 1974;120:458-65.

366 [21] Munger K, Germann UA, Lerch K. Isolation and structural organization of the Neurospora crassa copper metallothionein gene. The EMBO journal. 1985;4:2665-8.

368 [22] R Breathnach a, Chambon P. Organization and Expression of Eucaryotic Split Genes 369 Coding for Proteins. Annual Review of Biochemistry. 1981;50:349-83.

370 [23] Carattoli A, Cogoni C, Morelli G, Macino G. Molecular characterization of upstream 371 regulatory sequences controlling the photoinduced expression of the albino-3 gene of 372 Neurospora crassa. Molecular Microbiology. 1994;13:787-95.

373 [24] Moore MM. Genetic engineering of fungal cells. In: Doelle HW, Dasilva EJ, editors. 374 Biotechnology Ontario, Canada: EOLSS; 2007. p. 36-63.

375 [25] Desai SH, Rabinovitch-Deere CA, Fan ZL, Atsumi S. Isobutanol production from cellobionic 376 acid in Escherichia coli. Microbial cell factories. 2015;14.

377 [26] Li X, Chomvong K, Yu VY, Liang JM, Lin YP, Cate JHD. Cellobionic acid utilization: from 378 Neurospora crassa to Saccharomyces cerevisiae. Biotechnology for biofuels. 2015;8:120.

379 [27] Hildebrand A, Schlacta T, Warmack R, Kasuga T, Fan Z. Engineering Escherichia coli for 380 improved ethanol production from gluconate. J Biotechnol. 2013;168:101-6. 
384 Table 1. Strains used in this study.

\begin{tabular}{|c|c|c|}
\hline Strain & Genotype & Source \\
\hline FGSC 2489 & Wild type & FGSC \\
\hline $\begin{array}{lr}\text { F5 } & \Delta \text { mus-51 } \\
\Delta \text { ace-1 } & \Delta \text { cre-1 } \\
\Delta \text { ndvB } & \end{array}$ & $\begin{array}{l}\text { bgl-1::hph bgl-2::hph bgl-3::hph bgl-4::hph bgl- } \\
6:: \text { hph bgl-7::hph mus-51::six ace-1::six cre- } \\
1:: \text { six ndvB::six matA }\end{array}$ & $\begin{array}{l}\text { (Hildebrand, } \\
\text { Szewczyk et al. 2015) }\end{array}$ \\
\hline AH003 & $\begin{array}{l}\text { F5 } \Delta m u s-51 \text { Aace-1 } \Delta c r e-1 \Delta n d v B \text { csr-1::gpdA- } \\
\text { Nclac }\end{array}$ & This study \\
\hline HL04 & $\begin{array}{l}\text { F5 } \Delta m u s-51 \text { Aace-1 } \Delta c r e-1 \Delta n d v B \text { csr-1::gpdA- } \\
\text { sBalac }\end{array}$ & This study \\
\hline HL05 & $\begin{array}{l}\text { F5 } \triangle m u s-51 \text { Aace-1 } \Delta c r e-1 \Delta n d v B \text { csr-1::pcmt-1- } \\
\text { Nclac }\end{array}$ & This study \\
\hline HL10 & $\begin{array}{l}\text { F5 } \triangle m u s-51 \text { Aace-1 } \Delta c r e-1 \Delta n d v B \text { csr-1::pcmt-1- } \\
\text { sBalac }\end{array}$ & This study \\
\hline HL11 & $\begin{array}{l}\text { F5 } \Delta m u s-51 \text { Aace-1 } \Delta \text { cre-1 } \Delta n d v B \text { csr-1::pcmt- } \\
\text { Nclac }\end{array}$ & This study \\
\hline HL13 & $\begin{array}{l}\text { F5 Amus-51 } \Delta a c e-1 \text { Acre-1 } \Delta \text { ndvB csr-1::pcmt- } \\
\text { sBalac }\end{array}$ & This study \\
\hline
\end{tabular}


387 Table 2. Primers used in strain construction and verification.

\begin{tabular}{|l|l|}
\hline Primer & Sequence (5' to 3') \\
\hline NcLac-F & TCACCcacgtgATGAAATTCTTGGGCATTGCCG \\
\hline NcLac-R & cctgcaggtacgtaCTACCACTTCATCTTCACCTCCCTCG \\
\hline pcmt-slic-F & GTGTGCAGCGCAGGAAATCCAccgcggCATCATAAGATCATCACAAACCACCCA \\
\hline pcmt-1-slic-F & GTGTGCAGCGCAGGAAATCCAccgcggTAGTGTACGATGAGGGTATTTCGCC \\
\hline pcmt-slic-NcR & GCAATGCCCAAGAATTTCATcacgtgTTTGATAGAAGTGGTTGTTCTTTTGAGAGG \\
\hline pcmt-slic-sBaR & AACACGGTAAAGTACTTCATcacgtgTTTGATAGAAGTGGTTGTTCTTTTGAGAGG \\
\hline 3flank_slic_R & TGGATTTCCTGCGCTGCAC \\
\hline NcLac-F & ATGAAATTCTTGGGCATTGCCG \\
\hline sBaLac-F & ATGAAGTACTTTACCGTGTTCACCGCC \\
\hline sBaLac-R & TCAGTGGTGATGGTGGTGGTG \\
\hline
\end{tabular}


389 Table 3. Percentage of Avicel hydrolyzed and the percentage directed toward fermentable

390 products for the F5 $5 m u s-51 \Delta a c e-1 \Delta c r e-1 \Delta n d v B$ and HL10 strains grown on $20 \mathrm{~g} / \mathrm{L}$ Avicel.

391 Errors are calculated based upon standard deviations and error propagation theory.

\begin{tabular}{|c|c|c|c|c|c|c|}
\hline & $\begin{array}{l}\text { Starting } \\
\text { Avicel } \\
\text { (mg) }\end{array}$ & $\begin{array}{c}\text { Residual } \\
\text { Avicel } \\
\text { (mg) }\end{array}$ & $\begin{array}{c}\text { Cellulose } \\
\text { Conversion } \\
(\%)\end{array}$ & $\begin{array}{l}\text { Mycelium } \\
\text { produced } \\
(\mathrm{mg})\end{array}$ & $\begin{array}{c}\text { Yield of } \\
\text { cellobionate } \\
\text { and } \\
\text { cellobiose } \\
\text { from } \\
\text { consumed } \\
\text { Avicel } \\
\text { (mol/mol× } \\
100 \%)^{*}\end{array}$ & $\begin{array}{c}\text { Yield of } \\
\text { cellobionate } \\
\text { from } \\
\text { consumed } \\
\text { Avicel } \\
(\mathrm{mol} / \mathrm{mol} \times 1 \\
00 \%)^{*}\end{array}$ \\
\hline $\begin{array}{l}\mathrm{F} 5 \Delta m u s-51 \\
\Delta a c e-1 \Delta c r e-1 \\
\Delta n d v B\end{array}$ & 1000 & $305 \pm 6$ & $69.5 \pm 0.6$ & $90 \pm 1$ & $87.1 \pm 0.6$ & $39.9 \pm 0.3$ \\
\hline HL10 & 1000 & $188 \pm 3$ & $81.2 \pm 0.3$ & $81 \pm 5$ & $95.7 \pm 1.1$ & $94.5 \pm 0.9$ \\
\hline
\end{tabular}

392

$393 *$ Molecular weight of Avicel was assumed to be $324 \mathrm{~g} / \mathrm{mol}$. 
397 Figure 1. Laccase activities for the day 3 cultures of $N$. crassa recombinants with different

398 promoter and laccase gene cassettes insertion. Conditions: $N$. crassa recombinants were cultured 399 in 1x Vogel's media containing glucose $(40 \mathrm{~g} / \mathrm{L})$ and $\mathrm{CuSO}_{4}(0.5 \mathrm{mM})$ at $28^{\circ} \mathrm{C}$ and shaken at $400200 \mathrm{rpm}$ for 3 days. The values shown are the means of biological triplicates for each strain, and 401 the error bars are the standard deviations.

403 Figure 2. Laccase activities for the cultures of N. crassa HL10 (A) and N. crassa HL13 (B)

404 different copper adding time. Conditions: $N$. crassa was cultured in 1x Vogel's medium 405 containing glucose $(40 \mathrm{~g} / \mathrm{L})$ at $28^{\circ} \mathrm{C}$ and shaken at $200 \mathrm{rpm}$ with $\mathrm{CuSO}_{4}(0.5 \mathrm{mM})$ added at 406 different time. The values shown are the means of biological triplicates for each strain, and the 407 error bars are the standard deviations.

409 Figure 3. Laccase activities for the cultures of N. crassa HL10 (A) and N. crassa HL13 (B) at 410 different copper concentrations. Conditions: N. crassa was cultured in 1x Vogel's medium 411 containing glucose (40 g/L) and different concentration of $\mathrm{CuSO}_{4}$ at $28{ }^{\circ} \mathrm{C}$ and shaken at 200 $412 \mathrm{rpm}$. The values shown are the means of biological triplicates for each strain, and the error bars 413 are the standard deviations.

415 Figure 4. Laccase activities for the cultures of $N$. crassa HL10 at different culture temperatures. 416 Conditions: N. crassa was cultured in 1x Vogel's medium containing glucose (40 g/L) and $417 \mathrm{CuSO}_{4}(0.8 \mathrm{mM})$ at different temperature and shaken at $200 \mathrm{rpm}$. 
418 The values shown are the means of biological triplicates for each strain, and the error bars are the 419 standard deviations.

421 Figure 5. Cellobiose (A) and cellobionate (B) production by N. crassa HL10 in Avicel Vogel's 422 media at different fermentation temperature. Conditions: N. crassa was cultured in 1x Vogel's 423 medium containing Avicel (20 g/L), $\mathrm{CuSO}_{4}(0.8 \mathrm{mM})$ and ABTS (0.1 mM) at different 424 temperatures and shaken at $200 \mathrm{rpm}$. The values shown are the means of biological triplicates for 425 each strain, and the error bars are the standard deviations.

427 Figure 6. Exoglycanase activity (A), cellobiose and cellobionate production (B) by the F5 $\Delta$ mus$42851 \Delta$ ace- $1 \Delta$ cre- $1 \Delta \mathrm{ndvB}$ and HL10 strains grown on $20 \mathrm{~g} / \mathrm{L}$ Avicel. The values shown are the 429 means of biological triplicates for each strain, and the error bars are the standard deviations. 


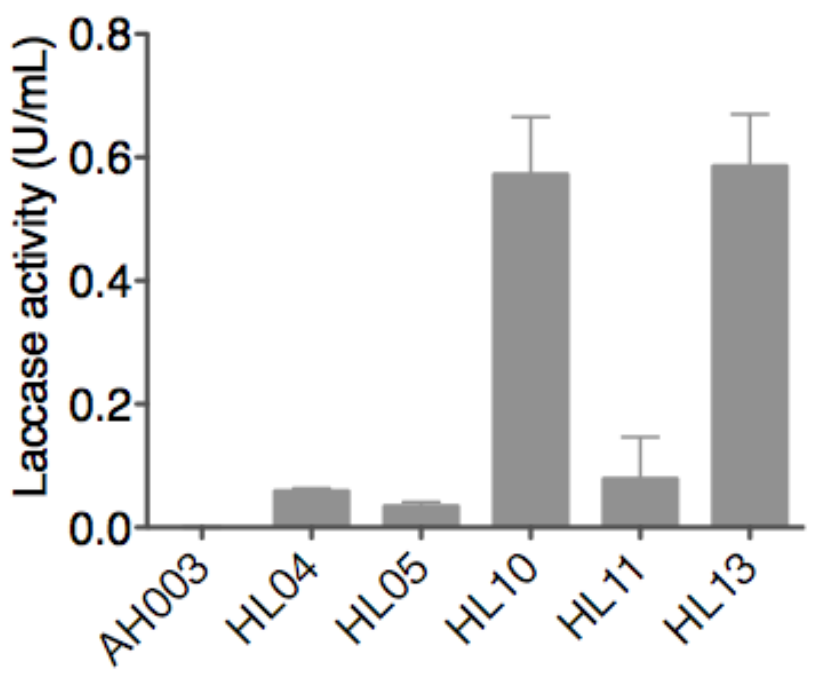

432

433 Figure 1

434 

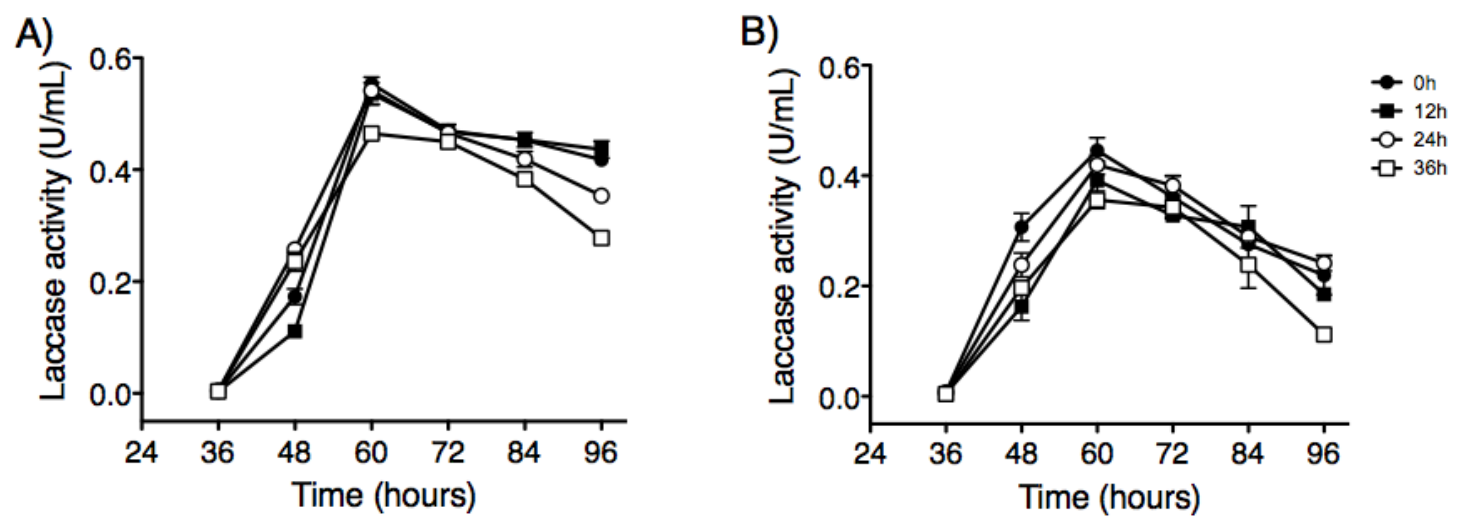

Figure 2. 

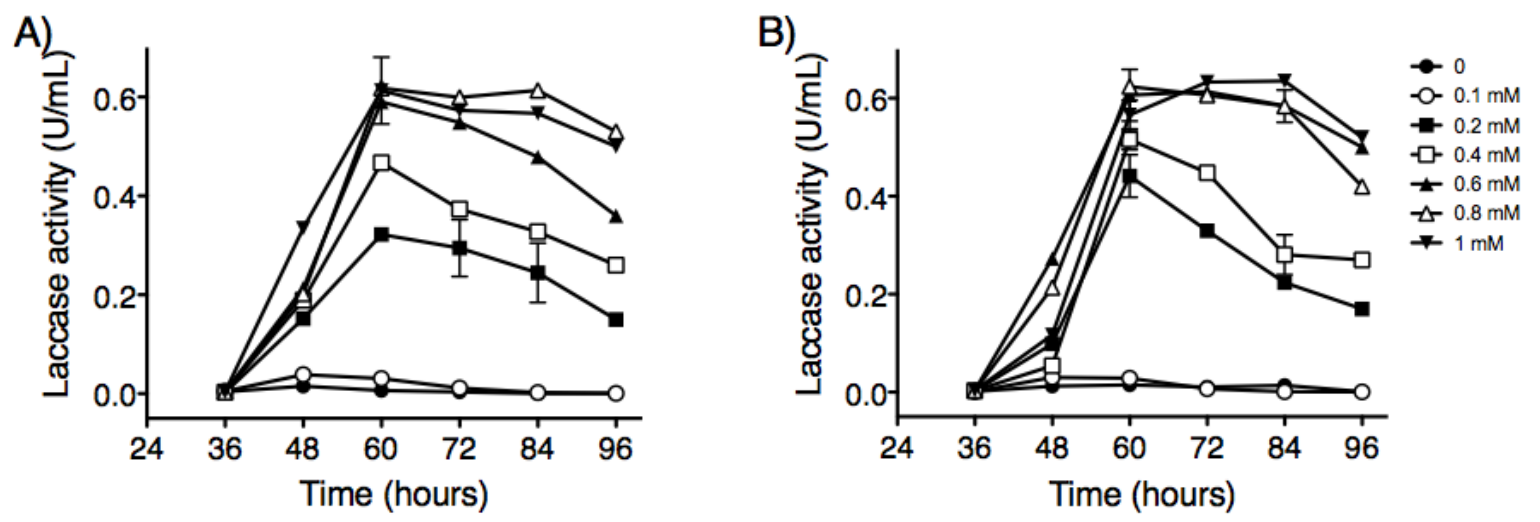

Figure 3 


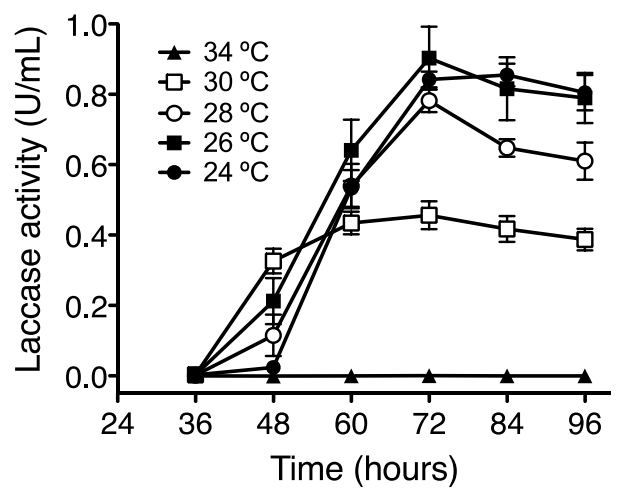

440

$441 \quad$ Figure 4.

442 

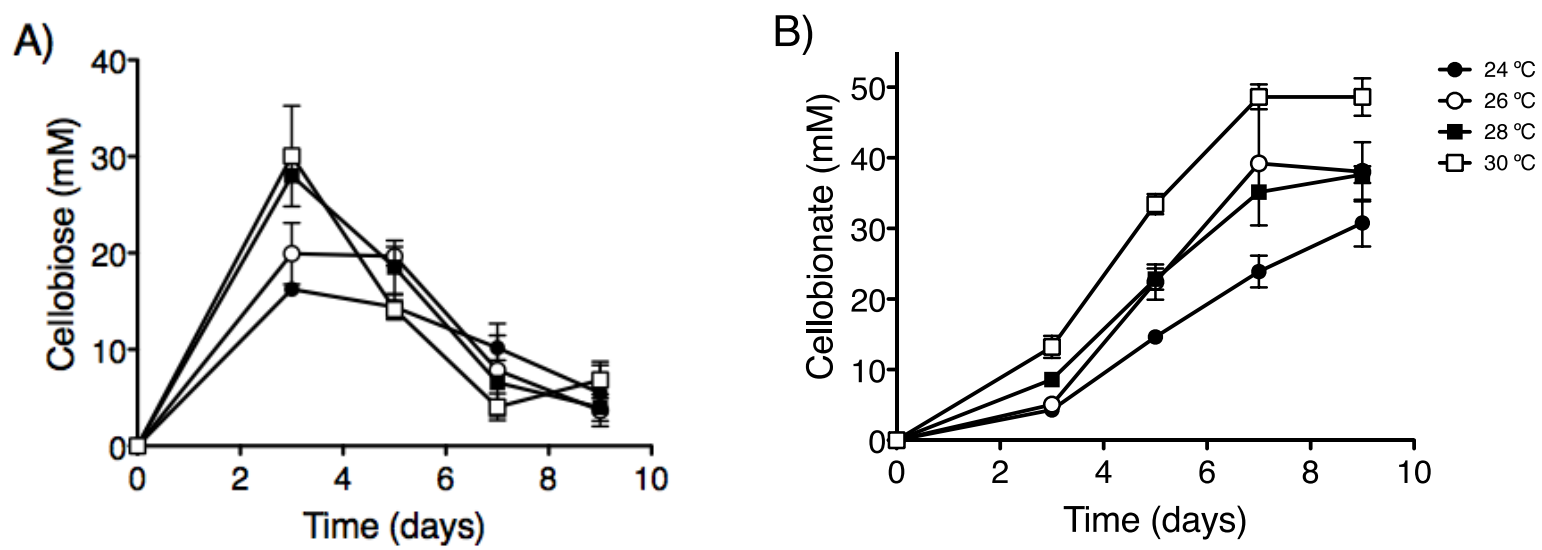

\section{Figure 5.}



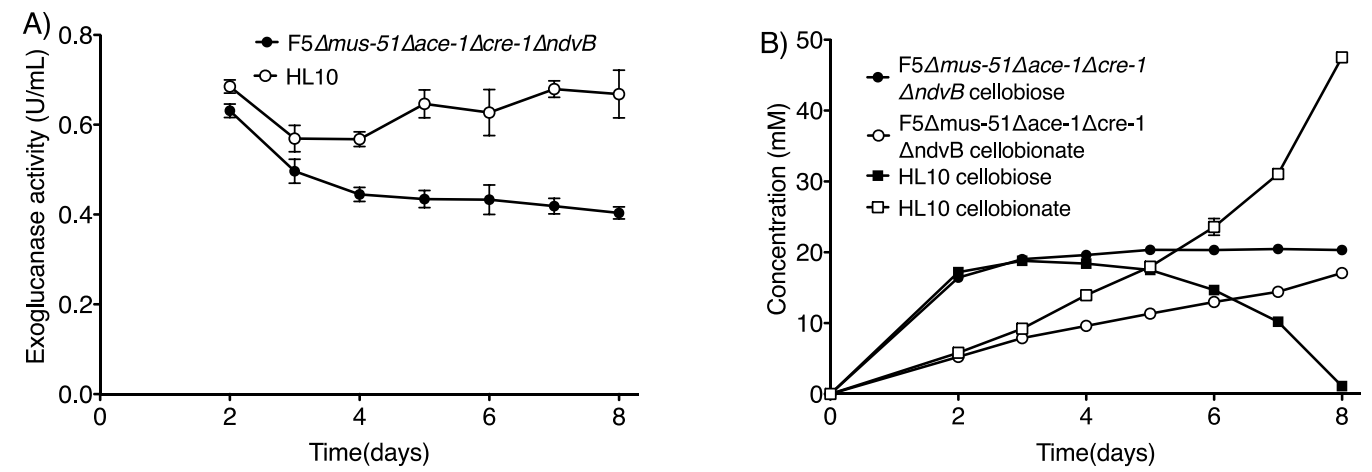

Figure 6 